\section{CASE REPORT}

E. Karaarslan

S. Ulus

M. Kürtüncü

\title{
Susceptibility-Weighted Imaging in Migraine with Aura
}

SUMMARY: We report 2 patients with migraine with aura, in whom SWI showed transient prominence of the venous vasculature within areas of impaired perfusion. The findings resolved spontaneously in both patients. This imaging technique may play a helpful role in assessing the vascular events in migraine with aura.

ABBREVIATIONS: $\mathrm{DWI}=$ diffusion-weighted imaging; FLAIR $=$ fluid-attenuated inversion recovery $\mathrm{MCA}=$ middle cerebral artery; $\mathrm{MRA}=\mathrm{MR}$ angiography; $\mathrm{MTT}=$ mean transit time; $\mathrm{rCBF}=$ relative cerebral blood flow; rCBV = relative cerebral blood volume; $\mathrm{SWI}=$ susceptibility-weighted imaging $\mathrm{TIA}=$ transient ischemic attack

M igraine is a common condition, classically presenting with unilateral debilitating painful headaches associated with photophobia, phonophobia, nausea, and vomiting. Migraine with aura typically presents with acute neurologic deficits, which may pose diagnostic difficulties. Acute ischemic cerebrovascular disease, vasculitis, focal infections, etc, must be considered in the differential diagnosis. ${ }^{1}$ In this article, we report 2 patients with migraines with aura with neuroradiologic imaging findings, including SWI.

\section{Case Reports}

\section{Patient 1}

A 37-year-old man presented with an acute-onset left homonymous hemianopsia preceding numbness in the right arm and face. A throbbing right-sided frontal headache followed 30 minutes after his neurologic deficit. At the time of his presentation to the emergency department, he had severe headache and nausea. His vital signs and the results of his routine blood biochemistry and hematologic work-up were normal. Findings of his physical and neurologic examinations were within normal limits except for a left homonymous hemianopsia. He was admitted to the neurology department with the initial diagnosis of migraine with aura. To rule out stroke, we performed cranial MR imaging, including SWI, DWI, perfusion MR imaging, and MRA. Routine T1-weighted images, T2-weighted images, and FLAIR images showed no abnormalities (not shown). DWI findings were also normal (not shown). MRA with 3D time-of-flight techniques revealed an indistinct appearance of the distal branches of the right MCA (Fig 1A). There was mild delay in the MTT and mild decrease in the $\mathrm{rCBV}$ and $\mathrm{rCBF}$ in the brain parenchyma of the right parieto-occipital lobe on the perfusion MR images (Fig 1B). SWI showed prominence of the venous vasculature in the region of the right parieto-occipital lobe (Fig 1C).

The patient's headache and neurologic deficit resolved completely after 10 hours. Two days after his attack, perfusion MR imaging, MRA (Fig $2 A$ ), and SWI (Fig $2 B$ ) were repeated; the findings were normal.

Received September 8, 2009; accepted after revision September 25.

From the Department of Radiology (E.K., S.U.) and Department of Neurology (M.K.), Acibadem University, Maslak, Istanbul, Turkey.

Please address correspondence to Ercan Karaarslan, MD, Acibadem Maslak Hastanesi, Büyükdere Cad. No. 40, 34457, Maslak, Istanbul, Turkey; e-mail: ercankaraarslan@ yahoo.com

DOI 10.3174/ajnr.A1973
He was discharged with the diagnosis of migraine with aura without any prophylactic treatment.

\section{Patient 2}

A 9-year-old boy presented to the emergency department with headache, numbness in the right arm, dysarthria, and right facial paresis. Results of blood pressure and routine blood biochemical and hematologic work-up were unremarkable. His neurologic examination revealed mild right facial paresis with normal funduscopy. He was admitted to the pediatric neurology department. Cranial MR imaging, including DWI and SWI, diffusion MR imaging, perfusion MR imaging, and intraextracranial MRA, was performed. Routine T1weighted images, T2-weighted images, and FLAIR images showed no abnormal findings (not shown). Findings of DWI were also normal (not shown). SWI showed prominence of the venous vasculature in the left parietal and occipital lobes (Fig $3 A$ ). There was mild delay in the MTT and mild decrease in the $\mathrm{rCBV}$ and $\mathrm{rCBF}$ in the brain parenchyma of the left parieto-occipital lobe on the perfusion MR imaging (Fig 3B)

His headache and neurologic deficit resolved completely within 12 hours. Two days after his admission, perfusion MR imaging and MRA were repeated, and the findings were all normal (not shown). He was discharged with the diagnosis of migraine with aura on flunarizine, $5 \mathrm{mg}$ per day.

\section{Discussion}

Migraine is a common condition that usually presents with unilateral debilitating headaches associated with photophobia, phonophobia, nausea, and vomiting. ${ }^{2}$ Less than $30 \%$ of migraine attacks are preceded by an aura, which is defined as any negative or positive neurologic symptom that usually lasts $<60$ minutes. The neurologic symptoms are mostly visual, but sensory and motor deficits may also occur, which can pose diagnostic difficulties such as the possibility of acute ischemic cerebrovascular disease. TIA was excluded because of gradual and increasing onset of neurologic symptoms and normal DWI findings in our patients. Other infectious and inflammatory diseases were also excluded by normal results of blood work-up and reversible imaging findings.

The pathophysiology of migraine is not completely understood, and there are basically 2 theories. The vascular theory suggests that the migraine aura is associated with vasoconstriction, and the subsequent headache is associated with vasodilation and hyperperfusion. ${ }^{3}$ The second theory pro- 

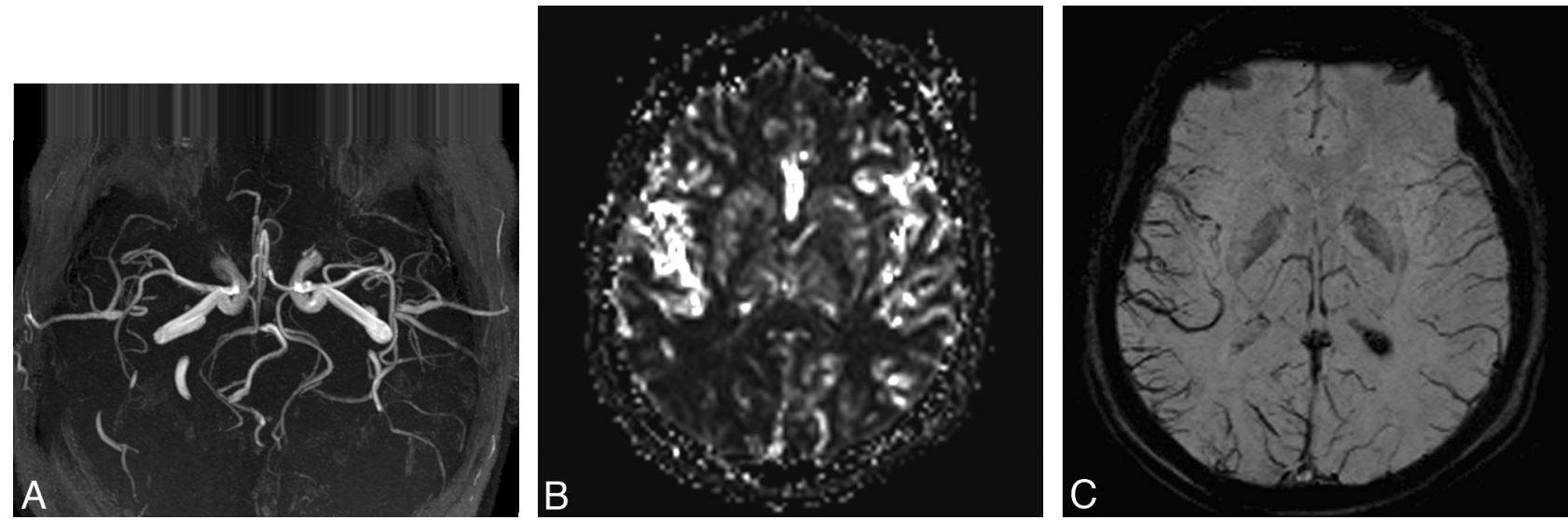

Fig 1. $A$, MRA shows the indistinct arteries of the right MCA. $B$, rCBF image shows impaired perfusion in the right parieto-occipital lobe. $C$, SWI reveals prominence of the venous vasculature in the region of the right parieto-occipital lobe.
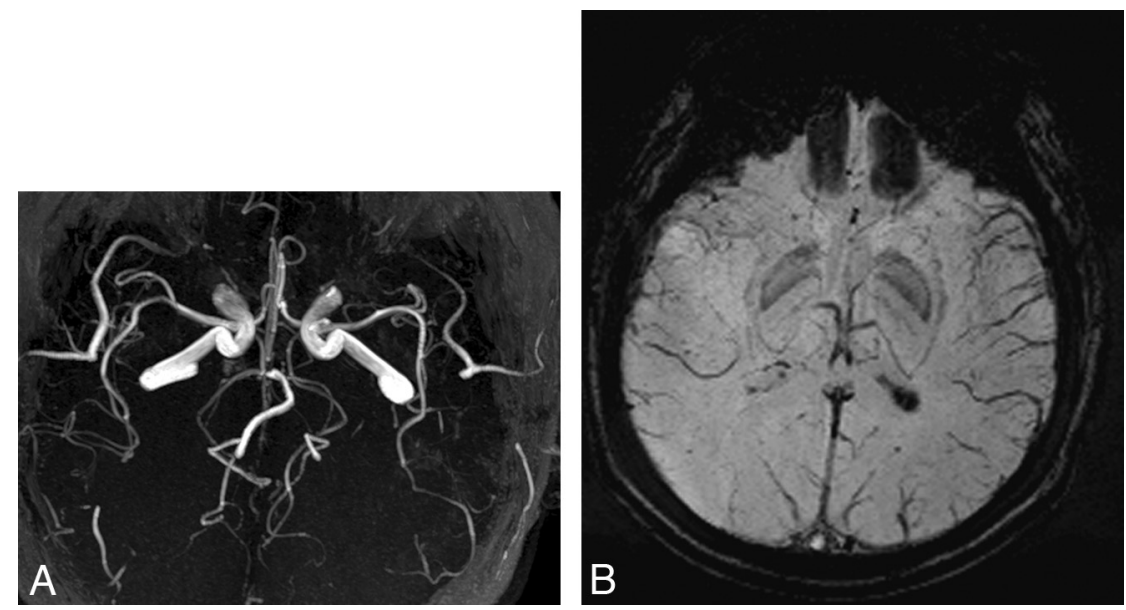

Fig 2. A, Normal findings on MRA 2 days after attack. $B$, Normal SWl finding 2 days after attack.
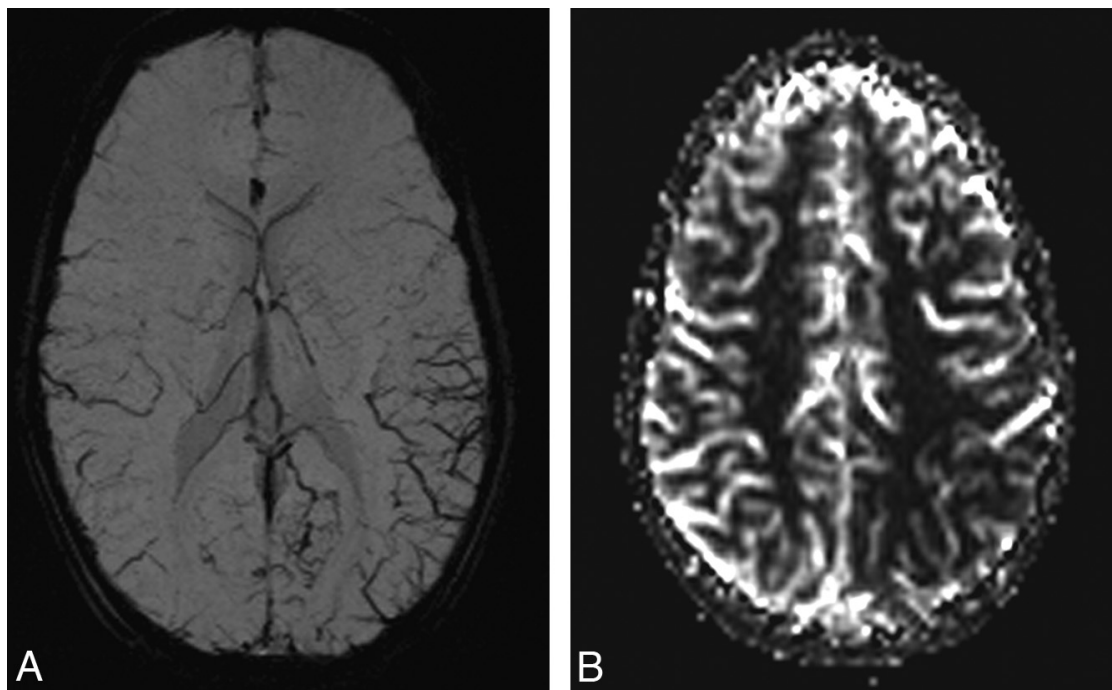

Fig 3. $A$, SWI reveals prominence of the venous vasculature in the region of the left parieto-occipital lobe. $B$, rCBF image shows impaired perfusion in the left parieto-occipital lobe.

poses that a contiguous wave of neuronal depression spreads through the cortex causing the aura symptoms. ${ }^{4,5}$ To understand the pathogenesis of a migraine headache, one must evaluate the changes in cerebral perfusion during the attack. Patients who undergo imaging examinations during an aura characteristically have hypoperfusion, whereas those who undergo imaging examinations during the headache phase show hyperperfusion. ${ }^{6}$ A mild dilation of the intracranial vessels contralateral to the hemiparesis has been reported. ${ }^{7,8}$ In our patients, the MR imaging studies were performed 
during the aura phase, and the MRA and perfusion MR imaging were consistent with this phase, revealing reversible vasoconstriction.

SWI is a fully velocity-compensated high-resolution 3D gradient-echo sequence used to create new sources of contrast. ${ }^{9}$ It provides additional clinically important information that can be complementary to conventional MR imaging sequences. In our patients, SWI showed prominent hypointense signals of the venous vasculature within areas of impaired perfusion. ${ }^{9,10}$ Uncoupling between oxygen supply and demand in hypoperfused tissue may cause a relative increase of deoxyhemoglobin levels and a decrease of oxyhemoglobin in the tissue capillaries and the draining veins. The areas with more prominent veins on SWI were in agreement with the abnormal area on the perfusion map.

Although the use of SWI may not be routinely recommended to assess migraine headaches, it may be helpful in the diagnosis of migraine with aura and may play a role in understanding the pathophysiology of migraine.

\section{References}

1. Schoene J, Sandor PS. Headache with focal neurological signs or symptoms: a complicated differential diagnosis. Lancet Neurol 2004;3:237-45

2. Headache Classification Subcommittee of the International Headache Society. The International Classification of Headache Disorders: 2nd ed. Cephalalgia 2004;24(suppl 1):9-160

3. Wolff HG. Headache and Other Head Pain. 2nd ed. New York: Oxford University Press; 1963

4. Lauritzen M. Cortical spreading depression in migraine. Cephalalgia 2001;21: 757-60

5. Goadsby PJ. Migraine pathophysiology. Headache 2005;45(suppl 1):S14-24

6. Pollock JM, Deibler AR, Burdette JH., et al. Migraine associated cerebral hyperperfusion with arterial spin-labeled MR imaging. AJNR Am J Neuroradiol 2008;29;1494-97

7. Butteriss DJ, Ramesh V, Birchall D. Serial MRI in a case of familial hemiplegic migraine. Neuroradiology 2003;45:300-03

8. Masuzaki M, Utsunomaiya $\mathrm{H}$, Yasumoto $\mathrm{S}$, et al. A case of hemiplegic migraine in childhood: transient unilateral hyperperfusion revealed by perfusion MR imaging and MR angiography. AJNR Am J Neuroradiol 2001;22:1795-97

9. Haacke EM, Mittal S, Wu Z, et al. Susceptibility-weighted imaging: technical aspects and clinical applications, part 2. AJNR Am J Neuroradiol 2009;30:23252. Epub 2009 Jan 8

10. Saini J, Thomas B, Bodhey NK, et al. Susceptibility-weighted imaging in cranial dural arteriovenous fistulas. AJNR Am J Neuroradiol 2009;30:E6 